Pirineos, 145-146: 81 a 89, JACA; 1995

\title{
VARIABILIDAD LONGITUDINAL DE LA ESCORRENTIA Y LA EROSION EN UNA LADERA QUEMADA
}

\author{
M. SOLER Y M. SALA \\ Departament de Geografia Física. Universitat de Barcelona. Baldiri Reixach s/n. 08028 Barcelona.
}

RESUMEN.- El objetivo de este estudio es observar las variaciones longitudinales de escorrentía y sedimento en una ladera quemada. La instrumentación utilizada se ha basado en los canales gerlach. La variación de precipitación así como algunos elementos del relieve, que pueden influir tanto en la escorrentía como en la erosión, también han sido estudiados.

No hay una relación directa entre la longitud del área de captación y el agua y el sedimento recogido en las trampas. No obstante, esta relación es positiva en los cuatro primeros metros de la ladera.

ABSTRACT.-The objective of this research is to study the variations of the runoff and erosion along a $29^{\circ}$ slope in a burnt area. The task has been done in Serra de Prades. Measurements have been undertaken with Gerlach troughs.

The variation of the erosion longitudinaly have been analized with Gerlach traps. The variation of precipitation and some elements of the relief which can influence runoff and erosion have also been studied. No direct relation has been found between the length of the capture of water and the amount of runoff and sediment collected in the traps. However this relation is positive in the first four metres of the slope.

Key-words: erosion, burnt area, Gerlach traps, Sierra de Prades (Spain).

\section{Introducción}

El objetivo de este trabajo es estudiar las variaciones a lo largo de la vertiente de la escorrentía y la pérdida de suelo en un área sometida a un incendio.

Horton (1940) fue de los primeros en proponer una teoría sobre la circulación de las aguas en las vertientes. En ella dice que la dinámica del agua cambia a lo largo de la vertiente, puesto que la potencia del flujo va aumentando ladera abajo a consecuencia de la acumulación del agua 
procedente de las partes altas. Autores posteriores han estudiado la hidrología de las vertientes en otras áreas y perciben que la hipótesis de Horton no siempre es válida. Dunne y Black (1970) tan solo confirman la escorrentía hortoniana en areas de: a) alta intensidad de precipitación, b) poca vegetación y c) ausencia de suelo con el sustrato a la vista. Yair (1985) establece que en zonas áridas la generación de escorrentía es más alta en relación a los afloramientos rocosos. Rose (1988) desarrolla un modelo para parcelas de uso agrícola donde relaciona la pérdida de material con la longitud de la parcela.

Las áreas afectadas por un incendio forestal presentan características de: a) ausencia de vegetación y b) escaso suelo. Por tanto podría ser que cumpliesen las predicciones de Horton.

Además, al carecer de suelo y de vegetación puede tener una respuesta similar a la de una parcela agrícola. Finalmente, también puede, durante un cierto tiempo, presentar características similares a las de una zona árida o semi-árida.

\section{Area de estudio}

El trabajo se ha realizado en la Sierra de.Prades (Tarragona), en una ladera a $900 \mathrm{~m}$ de altitud con pendientes de $29^{\circ}$ y orientación SSE. El substrato geológico está formado por pizarras poco metamórficas $y$ areniscas marinas de facies Culm (flysh) de edad Carbonífera. La precipitación media anual es de $600 \mathrm{~mm}$ y la vegetación predominante es la comunidad Quercus ilex sup. ilex de sotobosque empobrecido.

La selección de este área de estudio se debe a los diversos estudios pluridisciplinares que se llevan a término en este lugar desde el año 1977. basados en balances hidrológicos y de nutrientes a nivel de cuenca, así como la respuesta tanto física como química de un suelo sometido a un incendio (Escarré 1976).

\section{Diseño experimental}

Para llevar a término este estudio el diseño y la instrumentación son los siguientes:

Una parcela de $5 \mathrm{~m}$ de ancho por $40 \mathrm{~m}$ de largo donde se ha hecho una zanja en la parte superior para romper y desviar las aguas de drenaje. Se han instalado catorce canales apareados a , 2, 3, 4, 8, 9, 10 y $40 \mathrm{~m}$ de la zanja de tal forma que la superficie de captación de agua es de $2,3,4 ; 6$, 8 y $40 \mathrm{~m}$ (figura 1 ).

La precipitación se mide con 10 pluviómetros totalizadores instalados alrededor de la parcela; cinco de ellos están en posición vertical a un metro 
de altura y los otros cinco, a $30 \mathrm{~cm}$ del suelo y perpendiculares a la pendiente. La intensidad de precipitación se registra en un pluviógrafo situado a $2 \mathrm{Km}$ de la parcela. Para más detalles de la monitorización de las parcelas ver Soler \& Sala (1992).

La parcela se quemó el 28 de octubre de 1988. Los canales situados en la parte inferior se instalaron el mismo día y los de la parte superior en enero de 1989. Antes de la quema se talaron los árboles dejando las ramas de diámetro inferior a $2 \mathrm{~cm}$ sobre el suelo para conseguir una intensidad de fuego más alta. A pesar de esto la temperatura máxima alcanzada a 0.5 $m$ de altura fue de $600^{\circ} \mathrm{C}$ y en superfície de $300^{\circ} \mathrm{C}$ (Alcañiz et al 1989).

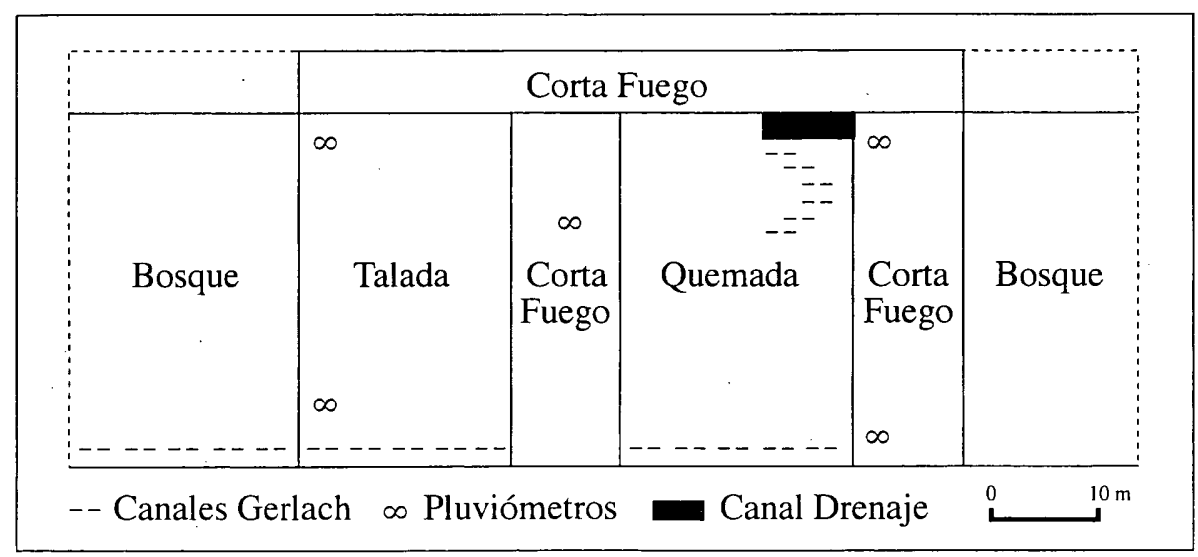

Fig. 1. Diseño experimental.

\section{Resultados}

\subsection{Variaciones longitudinales}

En la tabla 1 se observan los resultados de la pérdida de agua y sedimento obtenidos durante los 18 meses de estudio. Como se ha dicho anteriormente los canales están agrupados por parejas. Para hacer el tratamiento estadístico se ha calculado la media aritmética de estas parejas como si fuesen un único canal. Los resultados de la tabla 1 no intentan dar una tasa absoluta de erosión, pero sí es útil para establecer comparaciones $y$ dar un orden de magnitud.

Son cuatro los colectores que están situados a $4 \mathrm{~m}$ de longitud del área de drenaje, tal y como se observa en la figura 1. Los dos que están situados en la parte inferior captan el doble de sedimento y escorrentía respecto a los dos de la parte superior. 
PIRINEOS $145-146$

Tabla 1. Media de la escorrentía y sedimento en los 18 meses de estudio.

\begin{tabular}{|ccccccc|}
\hline LONG & AREA & $\begin{array}{c}\mathrm{Q} \\
(\mathrm{I})\end{array}$ & $\begin{array}{c}\mathrm{S} \\
(\mathrm{g})\end{array}$ & $\begin{array}{c}\mathrm{Q} \\
\left(1 \mathrm{~m}^{2}\right)\end{array}$ & $\begin{array}{c}\mathrm{S} \\
\left(\mathrm{g} \mathrm{m}^{2}\right)\end{array}$ & $\mathrm{n}$ \\
\hline 2 & 1 & 0.82 & 19.22 & 0.82 & 19.22 & 13 \\
3 & 1.5 & 2.63 & 33.56 & 1.75 & 22.37 & 13 \\
4 & 2 & 1.24 & 46.35 & 0.62 & 23.17 & 13 \\
6 & 3 & 1.12 & 135.41 & 0.37 & 45.13 & 13 \\
8 & 4 & 1.52 & 70.06 & 0.38 & 17.51 & 12 \\
40 & 20 & 4.14 & 78.16 & 0.21 & 3.90 & 13 \\
\hline
\end{tabular}

En cuanto al agua de escorrentía se ha ajustado en primer lugar una recta de regresión, para ver si hay una relación directa entre este agua y la zanja divisoria que rompe las aguas de drenaje. La ecuación de esta recta es de $y=0.96703-3.2084 e-2 x$, con $r^{2}=0.209$; por lo tanto la regresión es nula; realizado el test de Student da una probabilidad de hipótesis nula del 0.3106 . Si se ajustan los puntos a una curva logarítmica (figura 2) el coeficiente de relación mejora a $r^{2}=0.301$, pero continua siendo muy bajo.

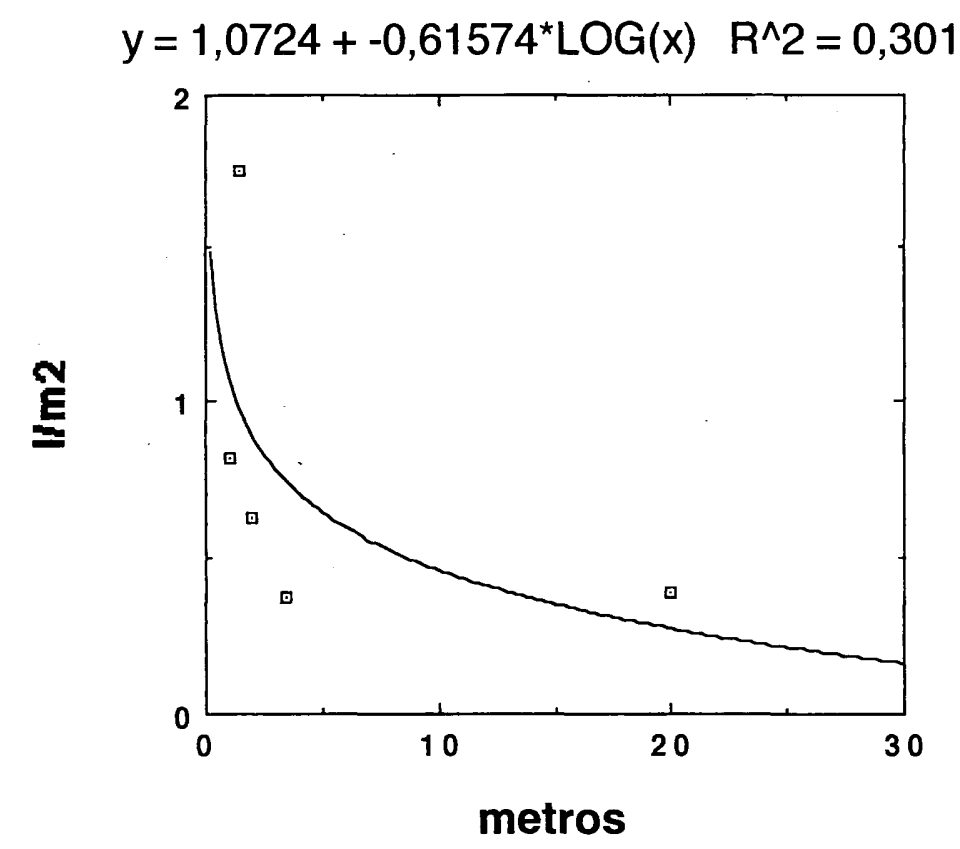

Fig. 2. Relación longitud/escorrentía. 
Por lo que se refiere al sedimento también se ha ajustado una recta de regresión cuya ecuación es de $y=27.773-0.41096 x$ con una $r^{2}=0.088$; realizado el test de Student da una probabilidad de hipótesis nula del 0.5284 . Sin embargo, si se saca el último punto (longitud del area de 20 m) la recta mejora con una $r^{2}=0.927$.

No obstante la relación del sedimento recogido en los gerlach, respecto a la zanja que se realizó para romper las aguas de drenaje se ajusta más a una función exponencial (figura 3 ), siempre sacando el último punto, cuya ecuación es de $y=12.763^{*} 10^{(0.1557 x)}$ con una $r^{2}=0.954$.

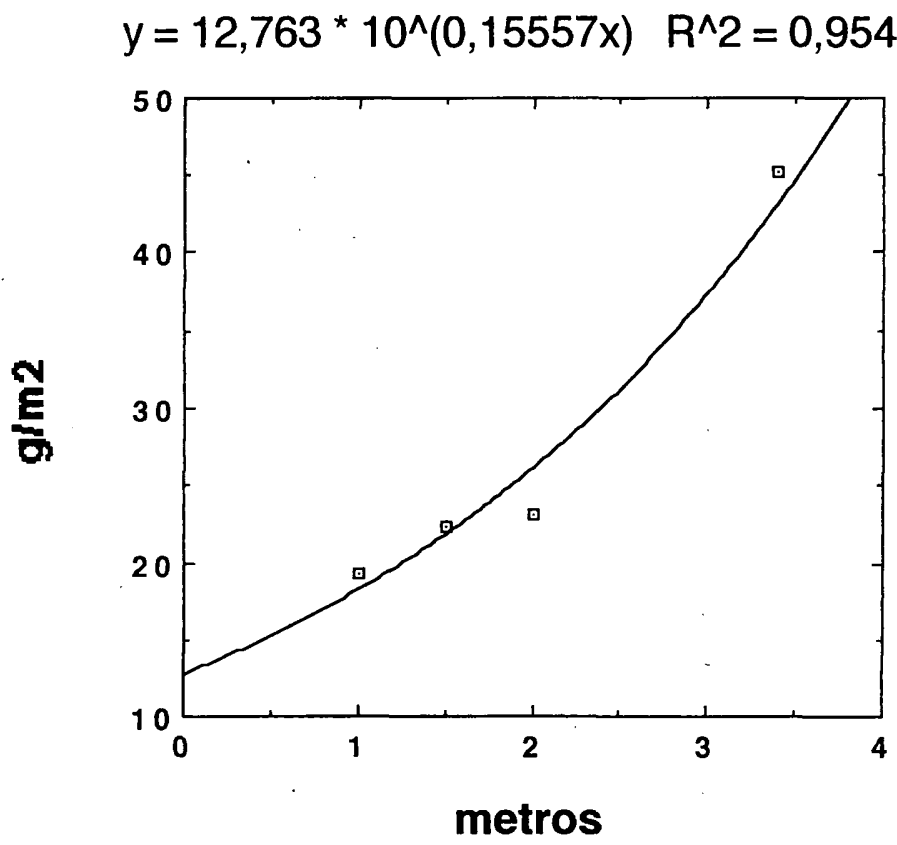

Fig. 3. Relación longitud/sedimentos.

Utilizando el modelo de Rose (1988), que establece una relación entre la pérdida de material y la longitud de la parcela, se observa que tanto en su modelo como con los datos reales de nuestra parcela hay un pequeño aumento de producción de sedimento vertiente abajo, pero este tiende a desaparecer hacia los 4 metros en nuestro caso y los 10 metros en el modelo de Rose (figura 4). 


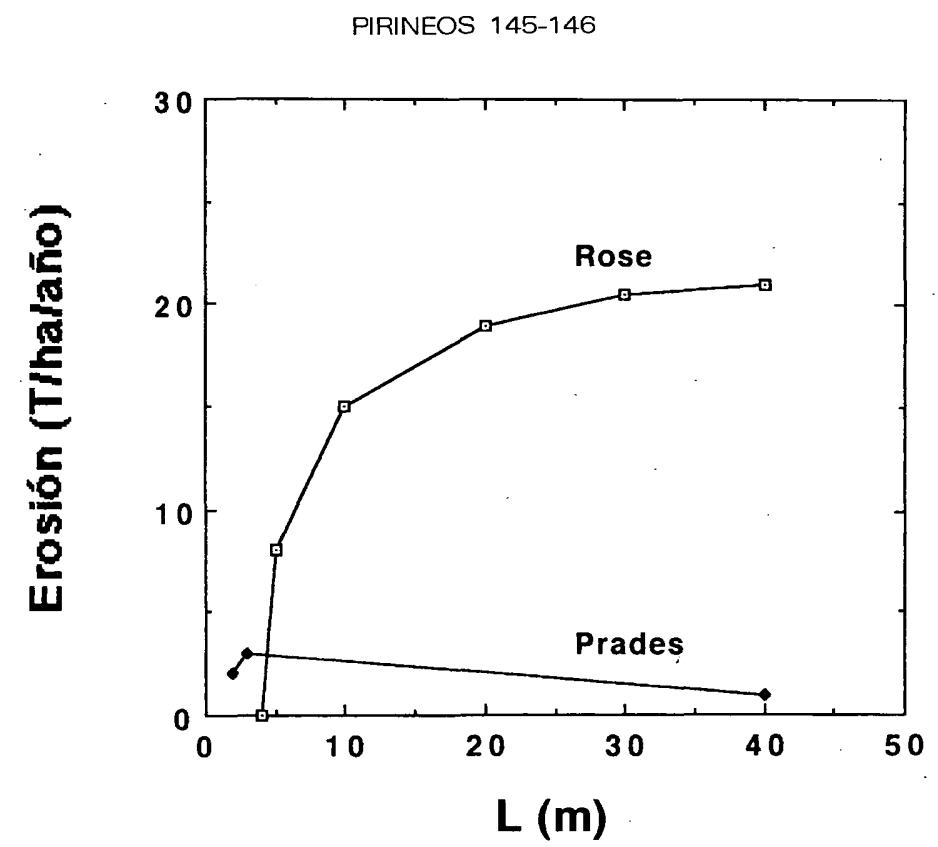

Fig. 4. Modelo Rose (1988)

\subsection{Variaciones en la precipitación}

Aparte de la longitud de la vertiente hay otros factores a considerar para establecer el recorrido del agua como la variación de la precipitación. Sharon (1980) observa que hay diferencias de precipitación a pequeñas escalas a causa de la dirección e inclinación de la lluvia y de la orientación e inclinación de la vertiente. Este aspecto, en la Sierra de Prades, no tiene mucha importancia. Ya se ha explicado la instrumentación y situación de los pluviómetros totalizadores en la parcela. En cuanto a la distribución y suponiendo que los que se encuentran situados en la parte baja de la vertiente recogen el $100 \%$ del agua de lluvia, los que están situados en la parte alta retienen un $92 \%$. Suponiendo que los que están a un metro de altura y en posición vertical caṕtan un $120 \%$ de la precipitación, los que están a $30 \mathrm{~cm}$ y perpendiculares a la vertiente recogen un $89 \%$, no obstante esta diferencia no es estadísticamente significativa: $P=0.6279$ (test Student).

\subsection{Relaciones con elementos del relieve post incendio}

Para comprender mejor la variabilidad de la pérdida de suelo dentro de la vertiente se han medido algunos elementos del suelo que comprenden

86 
ESTUDIO SOBRE LA VARIABILIDAD LONGITUDINAL

la microtopografía de la parte superior de la parcela quemada. Estos se han observado en un metro cuadrado aguas arriba de cada uno de los gerlachs. Se ha hecho por cada gerlach individualmente para obtener una mejor fiabilidad estadística, ya que si no habría pocos grados de libertad.

Con estos elementos se ha realizado una regresión múltiple paso a paso ascendente donde la variable a explicar es la pérdida de sedimento y las variables explicativas son: 1) distancia de cada gerlach a la zanja divisoria de las aguas de drenaje (longitud), 2) tanto por ciento de tocones, 3) pendiente local de cada canal, 4) tanto por ciento de predregosidad $y$ 5 ) sumatorio del agua de arroyada que ha sido recolectada en cada gerlach (tabla 2).

Tabla 2. Elementos del microrelieve.

\begin{tabular}{|ccccc|}
\hline $\begin{array}{c}\text { LONG } \\
(\mathrm{m})\end{array}$ & $\begin{array}{c}\text { TOCON } \\
(\%)\end{array}$ & $\begin{array}{c}\text { PEND } \\
(\mathrm{O})\end{array}$ & $\begin{array}{c}\text { PIEDRA } \\
(\%)\end{array}$ & \multicolumn{1}{c|}{$\begin{array}{c}\mathrm{O} \\
(\mathrm{I})\end{array}$} \\
\hline 2 & 22 & 20 & 25 & 2.46 \\
2 & 0 & 15 & 36 & 16.76 \\
3 & 4 & 18 & 32 & 34.14 \\
3 & 3 & 19 & 24 & 1.00 \\
4 & 2 & 30 & 30 & 35.53 \\
4 & 12 & 35 & 15 & 10.69 \\
4 & 0 & 25 & 27 & 9.82 \\
4 & 0 & 25 & 9 & 9.68 \\
6 & 3 & 27 & 20 & 7.83 \\
6 & 8 & 20 & 25 & 20.35 \\
8 & 7 & 35 & 28 & 24.40 \\
8 & 3 & 27 & 32 & 12.17 \\
\hline
\end{tabular}

Tabla 3. Combinaciones lineales.

\begin{tabular}{|lcc|}
\hline Variable & Coeficiente & Coef. Corre. Parcial \\
\hline metros & 7.050 & 0.2957 \\
$R=0.4277$ & & \\
RA2 $=8.7411$ & & \\
G. L. $=10$ & & \\
pendiente & -2.6413 & 0.0000 \\
metros & 9.2750 & 0.4054 \\
R $=0.5126$ & & \\
RA2 $=0.0785$ & & \\
G.L $=9$ & & \\
\hline
\end{tabular}


Cómo muestra la tabla 3, a pesar de que sea la longitud la variable más significativa, es decir, la longitud del área de captación de los gerlach, su coeficiente de correlación es bajo y por lo tanto poco significativo. Creemos que las variables escogidas son las correctas, pero el bajo grado de significación se debe a que los grados de libertad también lo son.

\section{Conclusión y discusión}

No hay una relación directa entre la longitud del área de captación (10 metros) y la cantidad de agua de escorrentía recogida en los depósitos. Hay una mejor relación estadística entre la recogida de sedimento y una pequeña parte de la vertiente ( 4 metros).

Así pues, no existe escorrentía hortoniana, a pesar de tratarse de una superficie en la que no ha quedado suelo y vegetación. No obstante, hay que tener en cuenta que al cabo de medio año fue regenerándose la vegetación

Ruiz-Flaño et al(1992) también encuentran una alta variabilidad dentro de una misma vertiente. Encuentran más agua de escorrentía en zonas pedregosas que en las cubiertas de vegetación, independientemente de su posición a lo largo de la vertiente.

Las diferentes cantidades de escorrentía y sedimento recogidos en los canales gerlach, dentro de una misma parcela, pueden ser debidos a condiciones microtopográficas como la pendiente, pedregosidad, concavidades, convexidades, etc.

Yair et al (1985) consideran un factor determinante para el cambio de escorrentía en superfície las propiedades de los materiales superficiales, ya que en medios áridos las variaciones de permeabilidad en superfície hacen aumentar las discontinuidades de la escorrentía. En el caso de Prades, la alta pedregosidad encontrada en la vertiente estudiada también puede influir en la discontinuidad del agua recogida a lo largo de la vertiente.

Como conclusión final podemos decir que dentro de las laderas hay una notable movilización de material, pero éste no va acumulándose ni aumentando ladera abajo una vez superados los primeros metros. Así pués, se tendría que hablar de formas de transporte y movilización de material a lo largo de las vertientes más que de formas de erosión o pérdida de material.

Agradecimientos. Este trabajo se ha realizado dentro el proyecto "Respuesta de pequeñas cuencas en ecosistemas mediterráneos: monitorización, experimentación y modelización", con cargo al programa LUCDEME. 


\section{Referencias}

Alcañiz, J., Arias, J., Josà, R., Serrasolses, I., Solé, A. \& Vallejo, R. (1989): Informe sobre el proyecto "El efecto del incendio forestal sobre el suelo de la Serra de Prades". Internal repport, ICONA 3-15.

Dunne, T. \& Black, R.D. (1970b): Partial area contributions to storm runoff in a small New England watershed. Water Resources Research 7: 1160-1172.

Escarré, E., Lledó, M., Bellot, J., Escaples, A., Seva, E., Rovira, A. \& Sánchez. J.(1986): Balance hídrico, meteorización y erosión en una pequeña cuenca de encinar medietrráneo (Proyecto LUCDEME). ICONA, Monografías 47: 57-115.

Horton, R.E. (1940): An approach towards a physical interpretation of infiltration capacity; Soil Sci. Soc. Am., 5: 399-417.

Rose, C.W. (1988): Research progress on soil erosion processes and basis for soil conservation practices. Soil Erosion Research Methods, Lal (ed): 119-139.

Ruiz-Flaño, P., Lasanta-Martínez, T. \& García-Ruiz, J.M. (1992): La variabilidad espacial de la producción de escorrentía y sedimentos como base para la gestión de campos abandonados. Actas de la l/ Reunión Nacional de Geomorfología: $221-230$

Sharon, D. (1980): The distribution of hydrologically effective rainfall incident on sloping ground. J. Hydrol., 46: 165-188.

Soler, M. \& Sala, M. (1992): Effects of fire and of clearing in a Mediterranean Quercus ilex woodland: An experimental approach. Catena, 19: 321-332.

Yair, A. \& Lavee, H. (1985): Runoff generation in arid and semi-arid zones. In Anderson, MG. \& Bur TP. Hydrological Forecasting, John Wiley \& Sons Ltd. London. 